\title{
An LBT view of the Andromeda's satellite galaxies
}

\author{
Felice Cusano $^{1, \star}$, Alessia Garofalo ${ }^{1,2}$, Gisella Clementini ${ }^{1}$, and Michele Cignoni ${ }^{3}$ \\ ${ }^{1}$ INAF - Osservatorio Astronomico di Bologna, Via Ranzani 1, I - 40127 Bologna, Italy \\ ${ }^{2}$ Dipartimento di Fisica e Astronomia, Universitá di Bologna, viale Berti Pichat, 6/2, I - 40127 Bologna, Italy \\ ${ }^{3}$ Dipartimento di Fisica, Universitá di Pisa, Largo Bruno Pontecorvo, 3, 56127 Pisa, Italy
}

\begin{abstract}
Results are presented on deep ( $V \sim 26.5 \mathrm{mag})$ time series observations of four dwarf spheroidal galaxies (dSphs) in the Andromeda (M31) complex, namely, And XIX, And XXI, And XXV and And XXVII, that we have observed with the Large Binocular Telescope (LBT). We discovered in these galaxies a total of over 200 RR Lyrae stars and 19 Anomalous Cepheids. We also characterised the stellar populations and the spatial distributions of these dSphs.
\end{abstract}

\section{Introduction}

$\Lambda$-CDM theory predicts the formation of massive galaxies like the Milky Way and M31 to occur by accretion and merging of smaller structures. However, some issues challenge this scenario like for instance, the missing satellites problem ([8]) or the observed alignment of satellite galaxies ([7]). The characterization of the resolved stellar populations and variable stars of the M31 satellites may allow to trace merging and accretion episodes occurred and still occurring in the M31 environment, thus helping to constrain the origin and the fate of Andromeda. We obtained $\mathrm{B}$ and $\mathrm{V}$ time-series photometry with the Large Binocular Cameras (LBC) of the LBT of four M31 dwarf satellite galaxies, namely, And XIX ([3]), And XXI ([4]), And XXV ([5]) and And XXVII ([6]).

\section{Observations and data reduction}

Observations of the four galaxies were obtained between 2010 and 2011. The LBCs were used in binocular mode and B images were acquired with the Blue camera of the LBC, whereas the V images with the Red LBC. PSF photometry was performed using the DAOPHOT-ALLSTAR-ALLFRAME package ([10]). Variable stars were identified using the variability index computed in DAOMASTER. The light curves of the candidate variables were then analyzed with the Graphical Analyzer of Time Series (GRATIS, [2]). Examples of light curves for RR Lyrae stars and Anomalous Cepheids (ACs) that we discovered in these galaxies are shown in Figure 1.

^felice.cusano@oabo.inaf.it 

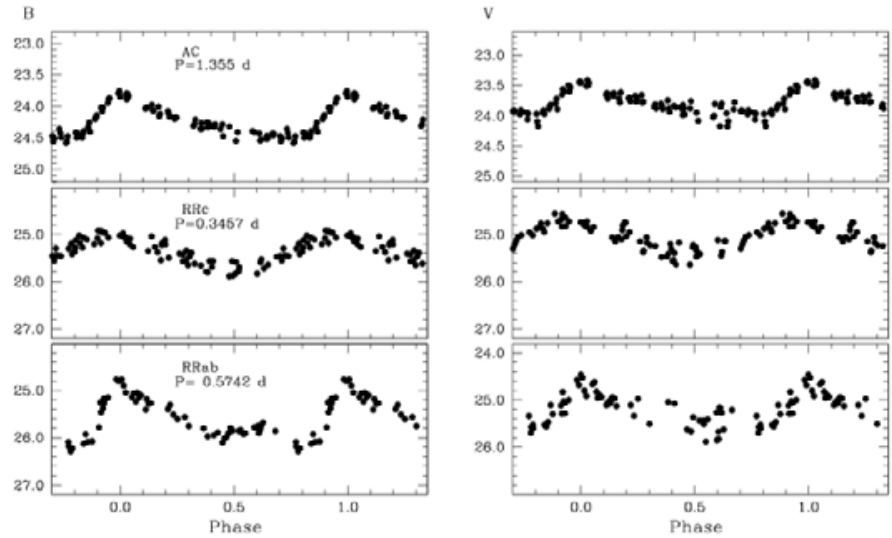

Figure 1. Examples of variable star light curves in And XXV. From top to bottom an AC, a first-overtone RR Lyrae star $(\mathrm{RRc})$ and a fundamental mode RR Lyrae star (RRab).

\section{Results}

We discovered a total of 218 RR Lyrae stars and 19 ACs in the four M31 satellites observed within our program. The properties of the variable stars are summarized in [6]. The M31 satellite galaxies that we imaged can be classified as Oosterhoff Intermediate (Oo-Int) systems ([1, 9]). There is a slight tendency towards Oo-I type with decreasing the distance to the M31. Besides identifying variable stars we also built color-magnitude diagrams (CMDs) to characterize the stellar populations of these M31 satellite galaxies. We found evidence for three different stellar populations in And XIX and And XXI: (1) an old $(\sim 12 \mathrm{Gyr})$ and metal-poor $([\mathrm{Fe} / \mathrm{H}]=-1.7 \mathrm{dex})$ component traced by the RR Lyrae stars; (2) a slightly younger $(10-6 \mathrm{Gyr})$ and more metal-rich $([\mathrm{Fe} / \mathrm{H}]=-1.5 \mathrm{dex})$ component populating the red horizontal branch, and (3) an intermediate age ( $\sim 1 \mathrm{Gyr})$ component with the same metallicity that produced the ACs. Instead, for And XXV and And XXVII we found indication of a single old star formation burst $(\sim 12 \mathrm{Gyr},[\mathrm{Fe} / \mathrm{H}]=-1.9 \mathrm{dex})$. The large field of view of the LBC allowed to study the spatial distribution of the different stellar populations in each galaxy. Isodensity contours coupled with the location of the RR Lyrae stars helped us to trace signatures of past and/or ongoing gravitational interactions with M31 or other dwarf galaxies. In particular: (a) we found evidence for And XXI to be the result of a past minor merger between two dwarf galaxies; (b) we discovered a spherically-shaped high density of stars near the And XXV center ([5]). This structure appears to be at a distance consistent with And XXV and we suggest it could either be a star cluster or the nucleus of And XXV; (c) we confirmed that And XXVII is a completely disrupted galaxy.

\section{References}

[1] Catelan, M., Ap\&SS, 320, 261 (2009)

[2] Clementini, G., Di Tomaso, S., Di Fabrizio, L., et al., AJ, 120, 2054 (2000)

[3] Cusano, F., Clementini, G., Garofalo, A., et al., ApJ, 779, 7 (2013)

[4] Cusano, F., Garofalo, A., Clementini, G., et al., ApJ, 806, 200 (2015)

[5] Cusano, F., Garofalo, A., Clementini, G., et al., ApJ, 829, 26 (2016)

[6] Cusano, F., Garofalo, A., Clementini, G., et al., ApJ, submitted (2017)

[7] Ibata, R. A., Lewis, G. F., Conn, A. R., et al., Nature, 493, 62 (2013)

[8] Moore, B., Ghigna, S., Governato, F., et al., ApJL, 524, L19 (1999)

[9] Oosterhoff, P. Th., The Observatory, 62, 104 (1939)

[10] Stetson, P. B., PASP, 106, 250 (1994) 